Ál varo Augusto Couto 1 Vanja Suely Calvosa 1 Raimundo Lacerda 1 Francisco Castro 1 Edval do Santa Rosa 1 José Maria Nascimento 1

\section{Controle da transmissão da malária em área de garimpo no Estado do Amapá com participação da iniciativa privada}

\author{
Control of malaria transmission in a gold-mining \\ area in Amapá State, Brazil, with participation \\ by private enterprise
}

1 Programa de Malária, Insti tuto Evandro Chagas, Fundação Nacional de Saúde. Av. Almirante Barroso 492, Belém, PA 66090-000, Brasil.
Abstract This paper reports on the epidemi ological characterization of malaria following implementation of a program to control the endemic in a gold-mining area in northern Amapá State. The study focuses on total malaria cases in Amapá and the impact of the disease on the population, as represented by the Mineração Novo Astro S/ A company and its employes as well as the community of Vila de Lourenço in the municipality of Calçoene, and adjacent gold mi ners. The effect of control measures in the program area is indicated by a significant reduction in malaria incidence and malaria-related morbidity and mortality. The importance of participation by private enterprise is emphasized, particularly in large projects for the control of endemic diseases (notably malaria) in the Amazon Region.

Key words Malaria; Communicable Disease Control; Prevention \& Control

Resumo Reportamos no presente trabalho a caracterização epi demiológica da malária, seguida da implantação de um programa de controle da endemia em uma área de garimpo, localizada ao norte do estado do Amapá.Consi dera-se a i nfluência desta região no número total de casos do estado eo impacto da doença na população representada pel os funcionári os e emprei tei ros da Mineração Novo Astro S/A, bem como pela comunidade da Vila de Lou renço, Muni cípio de Calçoene, Ama pá e garimpos adjacentes. O impacto das ações de controle adotadas no âmbito do programa, caracterizam-se pela significati va redução da inci dência da malária na área ea redução da morbi-mortalidade. Ressal ta-sea importância da participação da ini ciati va privada, principal mente aquelas que desenvolvem proj etos de grande portena região Amazônica brasileira, no controle das endemias prevalentes na região, particularmente a malária. Palavras-chave Malária; Controle de Doenças Transmissíveis; Prevenção \& Controle 
Introdução

A região Amazônica brasileira concentra quase que a totalidade dos casos de malária do Brasil. Em 1998, segundo os registros da Fundação Nacional de Saúde (FUNASA), foram reportados 471.892 casos de malária no país, sendo que 469.980 (99,6\%) ocorridos na Amazônia brasileira (MS, 1999; OPS, 1987).

A década de 70 passou a ser um marco histórico para a malária no Brasil. No primeiro ano deste período, foram notificados cerca de 52.000 casos, observando-se nos anos seguintes um considerável aumento. Além da elevação quantitativa, houve uma forte expansão territorial da doença na Amazônia, e, desta para outras regiões do país, de forma que ao final da década já se registravam mais de 400.000 casos (Marques, 1986, 1987; Marques et al., 1986).

Curiosamente, a malária não tem uma distribuição homogênea na região Amazônica, uma vez que concentra-se em determinadas áreas com características específicas. Marques, 1986, investigando os casos de malária oriundos da Amazônia, além de evidenciar o movimento migratório interno como fator responsável pela disseminação da doença na região, identificou as áreas de garimpos, projetos de colonização e agropecuários como os principais focos de manuten ção e disseminação da malária para a região Amazônica e desta para outras regiões do país.

No Brasil, a malária tem aumentado nas últimas décadas, apesar das constantes atividades desenvolvidas pelos serviços de controle de endemias dos órgãos governamentais. Esforços são feitos para estabelecer programas de controle, objetivando reduzir os efeitos da doença na população exposta.

Alguns poucos projetos da iniciativa privada, instalados na Amazônia brasileira, têm contribuído para amenizar os problemas causados pela malária na população ligada às suas atividades. Esta participação é da mais al ta importância e tem merecido o apoio de instituições vinculadas ao M inistério da Saúde (MS), que dão as orientações técnicas necessárias para o êxito de al guns destes programas.

A Mineração Novo Astro S/A (MNA), durante o período em que desenvolveu suas atividades no Município de Calçoene (Distrito de Lourenço), Estado do Amapá, estava sediada numa área de intensa atividade garimpeira com grande fluxo populacional, e nos seus primeiros anos de atividade, de 1985 a 1990, enfrentou sérios problemas face ao elevado nível de transmissão de malária entre os seus funcionários e prestadores de serviços. Este fato, contri- buiu para que a direção da empresa resolvesse, a partir de 1991, valorizar o problema. Assim, viabilizou a implantação de um programa de controle da doença que foi elaborado e executado com a orientação de pesquisadores do Instituto Evandro Chagas (IEC), de Belém, Pará e com a colaboração direta da FUNASA, Coordenadoria Regional do Estado do Amapá.

Esta iniciativa contou com recursos específicos dotados pela Empresa, destinados ao meIhoramento das instalações hospitalares (ambulatório, laboratório e serviços de controlee vigilância de vetores) além de investimentos em recursos humanos na ampliação do quadro de pessoal e treinamentos. Insumos, transportes e equi pamentos também foram disponibilizados, o que permitiu a estruturação do serviço, inclusive com atendimento da população residente na Vila de Lourenço e adjacência.

Em linhas gerais, o objetivo foi o de estabelecer um programa de controle da malária no sentido de reduzir a transmissão da doença na população representada por funcionários, empreiteiros e moradores da Vila do Lourenço. Para tanto, foram estabelecidas algumas metas, objetivando, inicialmente caracterizar a área, identificar os fatores de risco de maior relevância epidemiológica, aprimorar tecnicamente os profissionais ligados ao serviço médico-ambulatorial da empresa e finalmente, el eger as prioridades para o estabelecimento de um efetivo controle da transmissão da malária na área.

\section{Material e métodos}

\section{Área de estudo}

O Distrito de Lourenço está localizado em uma região montanhosa conhecida como Serra Lombarda, que possui uma orientação Noroeste-Sudeste e se destaca do relevo mais plano encontrado para o Sul. A área de estudo possui as seguintes coordenadas geográficas: $2018^{\prime}$ $17^{\prime \prime}$ de latitude (S) e 510 38' 09" de longitude (O); situa-se ao norte do Estado do Amapá e dista, em linha reta, $240 \mathrm{~km}$ da capital Macapá, com uma área de $280 \mathrm{~km}^{2}$ pertencendo ao $\mathrm{Mu}$ nicípio de Calçoene, Amapá. O clima é tropical chuvoso, possuindo precipitações anuais em torno de $3.069 \mathrm{~mm}$ e temperatura média mensal de $25,6 \circ \mathrm{C}$, média anual de $240 \mathrm{C}$, com máxima e mínima de $30 \circ \mathrm{C}$ e $18 \circ \mathrm{C}$, respectivamente. A umidade relativa do ar é sempre superior a $75 \%$ e a altitude de $305 \mathrm{~m}$ acima do nível do mar (Torresine et al., 1993).

A população tem um perfil muito instável face a intensa migração, peculiar em áreas de 
garimpos. Estimava-se no início da década de 80, uma população em torno de 10.000 habitantes. Durante o período de realização deste trabalho, oscilou entre 2.500 a menos de 1.000 habitantes nos últimos anos (FUNASA, 1996).

Nos cinco primeiros anos de atividades, a MNA lavrou e tratou o minério extraído a céu aberto. A partir de 1990, optou por um projeto de lavra subterrânea mecanizada. O controle ambiental foi sempre uma prioridade, pois além de atender às resoluções do Conselho Nacional de Meio Ambiente (CONAMA), implantou um programa de monitoramento ambiental. Devido às características dos efluentes na área de domínio da Empresa e principalmente, pelas áreas adjacentes serem remanescentes de atividades garimpeiras, foram monitorados nas coleções hídricas, os seguinte parâmetros: pH, mercúrio, cianeto, zinco, óleos e graxas e sólidos totais (Torresine et al., 1993).

Perfil epidemiológico da malária

O Distrito do Lourenço no início da década de 80 foi responsável por mais de $50 \%$ dos casos de malária no estado do Amapá. Posteriormente, em função das estratégi as de controle desenvolvidas na época pela FUNASA - Coordenadoria Regional do Estado do Amapá e pelo esvaziamento populacional, devido a escassez do minério a céu aberto, a contribuição do Lourenço no número total de casos do estado foi consideravelmente reduzida. Contudo, considerando que a população da área estava estimada em 1.800 habitantes, sua taxa de incidência era muito elevada, com Índice Parasitário Anual (IPA) de 188,88/ 1.000 habitantes. A isto somam-se: os registros de Lâminas deVerificação de Cura (LVC) positivos extremamente elevados; o fato dos casos de malária atendidos fora do Posto de Notificação (PN) da FU NASA que não foram considerados nas estatísticas, como por exemplo o Hospital Dr. Toyofiça Abe da MNA, que nos anos de 1991 e 1992 atendeu e tratou 142 e 133 casos, respectivamente. Portanto, apesar da redução quantitativa da malária no Distrito de Lourenço, avaliada pelo IPA, o quadro acima descrito sugere que a região permanecia como área de prioridade, segundo a própria definição do MS.

Considerando esses aspectos, fica evidente que o trabalho realizado pela FUNASA na região, no período em questão, não foi suficiente para reduzir a transmissão e dificultar a dispersão da malária para outras localidades do estado do Amapá e além fronteira.

No que concerne à área limite da Companhia, o quadro epidemiológico da malária, até a implantação do programa, não era diferente, uma vez que registraram-se, entre 1987 e 1990, anos que antecederam à implantação do Programa, taxas de incidência bem elevadas com IPA de 962,2; 354,8; 706, 7 e 478, 7/ 1.000 habitantes, respectivamente.

\section{Metodologia do programa}

Num primeiro momento, entre novembro de 1991 a maio de 1992, foram realizadas várias atividades objetivando:

a) Identificar, delinear e caracterizar epidemiológica e entomologicamente o foco de transmissão;

b) Estabelecer os níveis de controle a serem alcançados;

c) Selecionar as medidas de intervenção mais adequadas e potencialmente mais eficazes;

d) Estabelecer as estratégias para aplicação das medidas de intervenção e implantação de um sistema de monitoração e avaliação, e finalmente;

e) Promover o aprimoramento técnico dos profissionais envolvidos no serviço de saúde e saneamento da empresa.

O programa de controle da malária, delineado para a área de abrangência da MNA, foi implantado em quatro fases distintas, conforme descrição a seguir:

\section{- Fase 1 (novembro de 1991)}

a) Avaliação das medidas de controleem prática; b) Avaliação da eficácia dos esquemas de tratamento e acompanhamento de cura;

c) Avaliação do grau da eficácia da quimioprofilaxia com sal cloroquinado, medida em uso nos últimos dois anos como estratégia de controle na área (Pinotti, 1953).

- Fase 2 (fevereiro de 1992)

a) Curso de aprimoramento técnico e implantação de um serviço de vigilância, envolvendo aspectos epidemiológicos da malária, diagnóstico clínico-epidemiológico, tratamento e avaliação da resposta do Plasmodium falciparum às drogas antimaláricas, e finalmente a monitoração de pacientes em ambulatório.

\section{- Fase 3 (maio de 1992)}

a) Identifi cação e caracterização dos criadouros de mosquitos do gênero Anopheles;

b) Determinação da freqüên cia e distribuição dos vetores encontrados na área; 
c) Identificação e grau de infectividade dos vetores;

d) Seleção de medidas de controle vetorial.

- Fase 4 (agosto e novembro de 1992)

Finalmente, a quarta e última fase, corresponde as duas avaliações do programa de controle. Tiveram como objetivo observar a evolução e o impacto no perfil epidemiológico da malária determinados pelas medidas adotadas pelo programa.

Medidas de intervenção e controle

No decorrer da implantação do programa de controle foram sugeridas e adotadas as seguintes medidas de controle:

- Medidas de modificação ambiental , tais como, obras de aterro, drenagem, canalização, retificação e represamento de curso de águas e alagados, manejo da insolação e sombreamento de criadouros:

- Borrifação intra e peridomiciliar;

- Ultra Baixo Volume(UBV);

- Tratamento químico e biológico dos criadouros, adotando-se o uso de larvicidas (Temefós) e em al guns foram introduzidos peixes larvófagos; - Outras medidas foram consideradas, tais como, proteção individual visando evitar ou reduzir o contato vetor-homem, utilização de esquemas terapêuticos mais eficazes no tratamento dos casos positivos, entre outros.

Caracterização entomológica

- Formas adultas

a) Densidade Populacional: foram criadas, em vários pontos do estrato epidemiológico, estações de coletas periódicas para acompanhamento da evolução da população de anofelinos em um ciclo trimestral na área da MNA e imediações, Vila do Lourenço, garimpos e baixões nas proximidades, no período de 1992 a 1995;

b) Ocorrência e distribuição das espécies de anofelinos: foram realizadas capturas em diferentes pontos da área limite da Companhia e imediações para composição do inquérito entomológico, objetivando o conhecimento da fauna anofélica em contato com o homem;

c) Grau de exofilia e endofilia: observação das condi ções habitacionais da área da M NA, Vila do Lourenço e imediações para sistematizar as coletas das formas aladas no intra, peri e extra domicílio concomitantemente;

d) Período e horário de coleta: para determinação da atividade de picar, as coletas foram realizadas padronizadamente entre às $18 \mathrm{~h}$ e $21 \mathrm{~h}$, período considerado de maior atividade do anofelino, durante três dias consecutivos, por três capturadores no mesmo ponto de coleta.

e) Grau de infecção natural: foram utilizados o método tradicional de dissecção e observação direta do parasita e o teste de "Enzymelinked immunosorbent assay" - ELISA (Wirtz et al., $1985,1987)$ com anticorpos monoclonais específicos para Plasmodium falciparum ou Plasmodium vivax, ou ainda $P$. vivax (variante VK 247). Pelo método de dissecção foram observados o estômago e glândulas salivares para pesquisa de oocistos e esporozoítos, respectivamente. Enquanto que com o teste de ELISA, além da identificação da positividade, foram determinadas as espécies de parasitas presentes. Para ambos os métodos, foram consideradas as formas de coleta (intra, peri ou extradomicílio) e tipo de isca utilizada.

\section{- Formas imaturas}

a) Identificação dos criadouros: identificação e caracterização das principais coleções hídricas na área da M NA e imediações, quanto aos aspectos físico-químicos e da vegetação aquática marginal aos mesmos.

\section{Resultados}

Na avaliação das medidas de controle realizadas durante a primeira fase, identificaram-se falhas metodológicas que de imediato foram interrompidas ou reorientadas. Destacaram-se aquelas relativas às ações anti-vetoriais, utilização de esquemas terapêuticos impróprios e ineficazes e, principalmente nos procedimentos de diagnóstico laboratorial.

Com o aprimoramento do diagnóstico laboratorial e a introdução de novos esquemas de tratamento, cuja eficácia já havia sido comprovada no programa de Malária do Instituto Evandro Chagas, houve um impacto imediato na qualidade do serviço de atendimento ambulatorial. Na Tabela 1, estão resumidos os esquemas de tratamento adotados pelo Programa de Saúde da MNA a partir de 1992.

A ocorrência de casos de malária no estado do Amapá no período de 1985 a 1998 e a contribuição do Distrito de Lourenço em relação ao número total de casos do estado, estão sumariados na (Tabela 2). No primeiro ano desta série histórica evidencia-se uma contribuição de $43,8 \%$ no número total de casos notificados. Identifica-se, pela análise do IPA geral do estado, um agravamento na transmissão da malá- 
ria, particularmente nos períodos compreendidos entre os anos de 1988 a 1990 com o IPA variando entre 32.6 a 37.7/1.000 habitantes e 1994 a 1997, quando esse indicador registra uma variação entre 33.4 a 65,5/ 1.000 habitantes ( Tabela 2).

No estrato epidemiológico, representado pela área de abrangência da MNA, a si tuação era mais grave. No período de 1985 a 1990 registrou-se uma elevada incidência na população, com IPA de até 1.478,67 e 1.613,09/ 1.000 habitantes em 1990 e 1992, respectivamente. Após a implementação do serviço de controle da malária, ocorreu uma redução bastante significativa no registro geral de casos. Já em 1993 foram notificados 73 casos e, nos anos seguintes, 1994 e 1995, reduziu para 40 e 26, respectivamente (Tabela 3).

O afastamento do trabal ho por malária que era de oito dias, em média, segundo os registros hospitalares da MNA, caiu para 4,2 dias em 1991, embora o melhor índice tenha sido alcançado nos anos seguintes, 1992 a 1994 com 2.0, 1.9 e 2.7 dias, respectivamente. A ocorrência de casos graves praticamente não existiram mais, exceto em atendimentos externos, fato que refletiu na redução significativa de internações hospitalares e conseqüentemente dimi-
Tabela 1

Esquemas de tratamento para malária causada por Plasmodium vivax e Plasmodium falciparum, adotado pelo Programa de Saúde da Mineração Novo Astro S/A, 1992-1995.

\begin{tabular}{|c|c|}
\hline Dias de tratamento & Tratamento \\
\hline \multicolumn{2}{|l|}{ Plasmodium vivax } \\
\hline Do & $4 \mathrm{cp}$ de Cloroquina $+1 \mathrm{cp}$ de Primaquina \\
\hline D1 a D2 & $3 \mathrm{cp}$ de Cloroquina $+1 \mathrm{cp}$ de Primaquina \\
\hline D3 a D13 & $1 \mathrm{cp}$ de Primaquina \\
\hline \multicolumn{2}{|c|}{ Plasmodium falcip arum } \\
\hline Nível I & Amodiaquina $(150 \mathrm{mg})+$ Tatraciclina $(500 \mathrm{mg})^{*}$ \\
\hline D0 & $4 \mathrm{cp}$ de Amodiaquina $+2 \mathrm{cp}$ de Tetraciclina $12 / 12 \mathrm{~h}$ \\
\hline D1 a D2 & $3 \mathrm{cp}$ de Amodiaquina $+2 \mathrm{cp}$ de Tetraciclina $12 / 12 \mathrm{~h}$ \\
\hline D3 a D6 & $2 \mathrm{cp}$ de Tetraciclina $12 / 12 \mathrm{~h}$ \\
\hline Nível II & Sulfato de Q uinino $(500 \mathrm{mg})+$ Tetraciclina $(500 \mathrm{mg})$ \\
\hline D0 a D2 & $2 \mathrm{cp}$ de $Q$ uinino $12 / 12 \mathrm{~h}+1 \mathrm{cp}$ de Tetraciclina $8 / 8 \mathrm{~h}$ \\
\hline D3 a D6 & $1 \mathrm{cp}$ de Tetraciclina $8 / 8 \mathrm{~h}$ \\
\hline Nível III & Sulfato de Quinino $(500 \mathrm{mg}$ ) \\
\hline D0 a D9 & $2 \mathrm{cp}$ de $Q$ uinino $12 / 12 \mathrm{~h}$ \\
\hline Nível IV & Mefloquina (250mg) \\
\hline D0 & $4 \mathrm{cp}$ de Mefloquina (DU) \\
\hline
\end{tabular}

* Esquema introduzido em função da baixa resistência do Plasmodium falciparum à Amodiaquina, determinada por testes de drogas in vitro, na área de estudo (Calvosa et al., 1992; Couto et al., 1995).

DU = dose única; $c p=$ comprimidos

Casos de malária no Estado do Amapá e contribuição do Distrito de Lourenço, Município de Calçoene, no total de casos do estado, 1985/1998.

\begin{tabular}{rrrrrrrrrrr}
\hline Anos & LE & Pf & Pv & Pm & Pf+Pv & Total & IPA & LOU & \% \\
\hline 1985 & 26.063 & 3.468 & 3.969 & 0 & 24 & 7.461 & 29,8 & 3.267 & 43,8 \\
1986 & 35.761 & 3.849 & 4.330 & 0 & 40 & 8.219 & 31,0 & 2.677 & 32,6 \\
1987 & 27.788 & 3.117 & 4.272 & 0 & 89 & 7.478 & 27,3 & 1.198 & 16,0 \\
1988 & 35.344 & 4.316 & 5.198 & 0 & 61 & 9.575 & 32,6 & 2.018 & 21,0 \\
1989 & 38.064 & 4.896 & 6.190 & 0 & 70 & 11.156 & 37,7 & 657 & 5,9 \\
1990 & 36.773 & 4.362 & 6.272 & 0 & 43 & 10.677 & 35,6 & 624 & 5,9 \\
1991 & 31.960 & 3.411 & 5.642 & 3 & 37 & 9.093 & 29,3 & DNC & DNC \\
1992 & 30.747 & 2.379 & 4.568 & 2 & 30 & 6.969 & 21,3 & DNC & DNC \\
1993 & 25.937 & 1.276 & 3.714 & 8 & 22 & 5.020 & 14,1 & DNC & DNC \\
1994 & 42.594 & 1.771 & 10.457 & 4 & 22 & 12.254 & 33,4 & 115 & 0,9 \\
1995 & 6.554 & 2.650 & 14.326 & 4 & 51 & 17.031 & 46,4 & 283 & 1,7 \\
1996 & 67.063 & 2.272 & 16.608 & 18 & 32 & 18.930 & 48,5 & 176 & 0,9 \\
1997 & 78.079 & 1.557 & 21.714 & 5 & 26 & 23.302 & 65,5 & 582 & 2,3 \\
1998 & 73.449 & 1.837 & 19.310 & 6 & 29 & 21.182 & 50,3 & DNC & DNC
\end{tabular}

Fonte: FUNASA, Coordenadoria Regional do Amapá.

$\mathrm{LE}=$ Lâminas Examinadas; Pf = Plasmodium falciparum;

$\mathrm{Pv}=$ Plasmodium vivax; $\mathrm{Pm}=$ Plasmodium malariae;

IPA = Índice Parasitário Anual/1.000 habitantes;

LOU = Distrito de Lourenço; DN C = Dados não conhecidos. 
Casos de malária na área de abrangência da Mineração Novo Astro S/A entre funcionários e empreiteiros, $1985-1995$.

\begin{tabular}{|c|c|c|c|c|c|c|c|c|c|c|}
\hline Anos & População & Casos (n) & $\begin{array}{l}\text { Média } \\
\text { mensal }\end{array}$ & IPA & $\begin{array}{c}\text { Casos C } \\
\text { Pf }\end{array}$ & $\begin{array}{l}\text { afast } \\
\text { Pv }\end{array}$ & $\begin{array}{l}\text { mento } d \\
P f+P v\end{array}$ & $\begin{array}{l}\text { trabalho } \\
\text { Total }\end{array}$ & Dias (n) & $\begin{array}{l}\text { Média por } \\
\text { tratamento }\end{array}$ \\
\hline 1985 & SI & SI & SI & SI & SI & SI & SI & SI & SI & SI \\
\hline 1986 & $\mathrm{SI}$ & $\mathrm{SI}$ & $\mathrm{SI}$ & SI & SI & $\mathrm{SI}$ & SI & SI & SI & $\mathrm{SI}$ \\
\hline 1987 & 688 & 662 & 55,2 & 962,21 & $\mathrm{SI}$ & $\mathrm{SI}$ & SI & SI & SI & SI \\
\hline 1988 & 1.071 & 380 & 31,7 & 354,81 & $\mathrm{SI}$ & $\mathrm{SI}$ & SI & SI & $\mathrm{SI}$ & $\mathrm{SI}$ \\
\hline 1989 & 341 & 241 & 20,1 & 706,74 & $\mathrm{SI}$ & $\mathrm{SI}$ & SI & SI & $\mathrm{SI}$ & $\mathrm{SI}$ \\
\hline 1990 & 211 & 312 & 26,0 & $1.478,67$ & SI & SI & SI & SI & $\mathrm{SI}$ & $\mathrm{SI}$ \\
\hline 1991 & 426 & 219 & 18,2 & 514,08 & 45 & 107 & 1 & 153 & 650 & 4,2 \\
\hline 1992 & 168 & 271 & 22,6 & $1.613,09$ & 30 & 95 & 0 & 125 & 259 & 2,0 \\
\hline 1993 & 403 & 73 & 6,1 & 181,14 & 9 & 24 & 0 & 33 & 69 & 1,9 \\
\hline 1994 & 403 & 40 & 3,3 & 99,25 & 3 & 10 & 1 & 14 & 38 & 2,7 \\
\hline 1995* & 337 & 26 & 4,3 & 77,15 & SI & SI & SI & SI & $\mathrm{SI}$ & SI \\
\hline
\end{tabular}

Fonte: Programa de Saúde da Mineração N ovo Astro S/A.

IPA = Índice Parasitário Anual; Pf = Plasmodium falciparum; Pv = Plasmodium vivax; SI = sem informação.

* N úmero de casos até o mês de junho.

nuindo o absenteísmo, que de 650 dias em 1991 caiu para 38 no ano de 1994 (Tabela 3).

Os registros do serviço de vigilância de cura constata o aumento de cura radical pela diminuição de LVC positivas, o que pressupõe a redução na ocorrência de recaí das e recrudescências.

Por ser considerada medida imprópria para região endêmi ca de malária e sua associação com a eclosão de cepas de P. falci parum resistentes, foi orientada a supressão da aplicação do sal cloroquinado, método que estava sendo utilizado como medida quimi oprofilática durante os últimos dois anos. Essa iniciativa parece ter sido responsável pela el evação de casos verificados no primeiro trimestre de 1992, em relação ao mesmo período do ano anterior.

Encontram-se na Tabela 4 dados referentes às coletas de larvas de anofelinos realizadas no período de maio a novembro de 1992. Na oportunidade foram observadas as características dos criadouros existentes na área da MNA e outros localizados nas proximidades. Foram classificados quanto aos componentes físicos, químicos e biológicos e identificados como tipos (I, II I II I IV eV). Finalmente, dependendo da extensão, foram demarcados pontos fixos de coleta, variando de 01 a 06 por criadouro.

No primeiro mês de coleta (maio/92), evidenciou-se que no principal e mais extenso criadouro (Lagoa do Lataia), foram coletadas 337 larvas, sendo que 94 (27.9\%) eram de Anopheles darlingi. Nos meses de agosto e novembro, já com reorientação das ações de controle vetorial, esse quadro foi se modificando e, pos- teriormente, o índice de positividade se estabilizou em níveis de controle.

O procedimento de captura de mosquitos adultos, também realizado no mesmo período, evidenciou a captura de 221 exemplares, predominantemente no peri-domicílio (112), no extra-domicílio (97) e intra-domicílio (12), conformeTabela 5 .

O A. darlingi foi a espécie mais freqüente nos três ambientes de captura, no intra-domicílio (100\%), peri-domicílio (91.1\%) e extra domicílio (89.3\%), bem como nas três estações de coleta, maio (98.0\%), agosto (69.9\%) e novembro (88.0\%), como mostra a Tabela 6.

A pesquisa para identificação da infectividade dos espéci mes capturados durante as três estações, revel ou positividade apenas para o A. darlingi com P. vivaxe P. vivax-vk247. O grau de infectividade, em maio, foi de $14.9 \%$ e nas estações seguintes, agosto e novembro foi de $6.4 \%$ e $4.0 \%$, respectivamente, mostrado na Tabela 6 .

\section{Discussão}

Na maioria dos países onde a malária tem um perfil endêmico os objetivos dos programas de controle da doença estão centrados na prevenção da mortalidade e na redução da morbidade, além da observância das perdas sócio-econômicas provocadas pela doença. Estas são as orientações resultantes da Conferência Ministerial Sobre Malária, real izada em Amsterdam no ano de 1992 (WHO, 1993). Essa estratégia, fundamentalmente, tem como premissa o diag- 
Freqüência de larvas de anofelinos coletadas nos criadouros da área de abrangência da Mineração Novo Astro S/A, no período de maio a novembro de 1992.

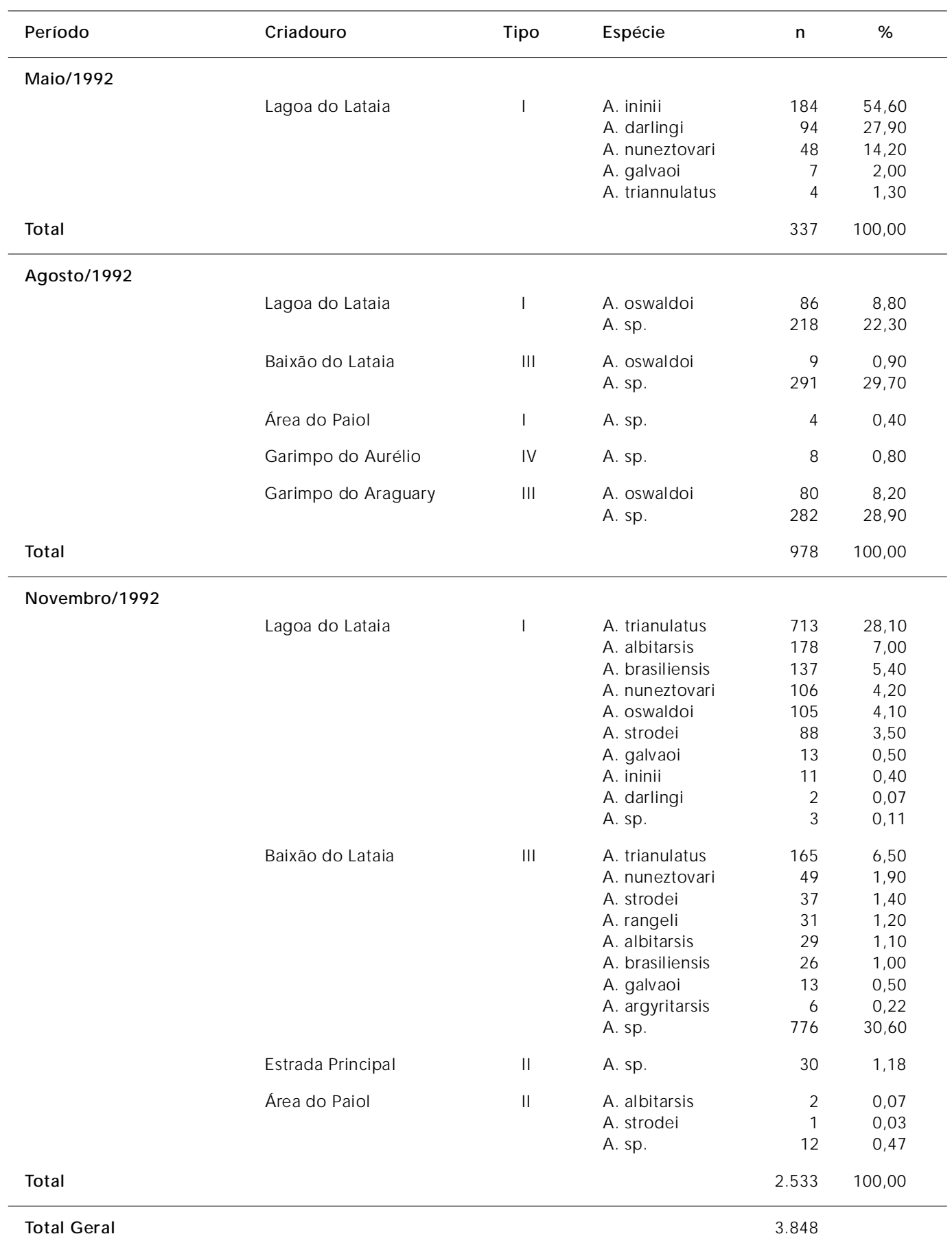

Fonte: Programa de Saúde da Mineração Novo Astro S/A.

Tipo I: Criadouro permanente formado por lagoas e escavações, sem correnteza, água turva,

vegetação vertical, pobre em matéria orgânica e ampla insolação.

Tipo II: Criadouro permanente formado por lagoas, sem correnteza, água límpida,

vegetação vertical, rico em matéria orgânica e média insolação.

Tipo III: Criadouro permanente formado por lagoas e escavações sem correnteza,

água límpida, vegetação vertical, rico em matéria orgânica e alta insolação.

Tipo IV: Criadouro permanente formado por lagoas e canais, com correnteza, água turva,

vegetação vertical, rico em matéria orgânica e média insolação.

Tipo V: Criadouro permanente formado por lagoas e canais, sem correnteza, água límpida,

vegetação vertical, rico em matéria orgânica e média insolação. 
Tabela 5

Captura de anofelinos na área de abrangência da Mineração Novo Astro S/A, no perío do de maio a novembro de 1992 , entre 18 e $21 \mathrm{~h}$.

\begin{tabular}{|c|c|c|c|c|c|c|}
\hline Período & Localização & Ponto & $\begin{array}{c}\text { Número } \\
\text { Intra }\end{array}$ & $\begin{array}{c}\text { de ex } \\
\text { Peri }\end{array}$ & $\begin{array}{c}\text { emplares } \\
\text { Extra }\end{array}$ & Espécies \\
\hline \multicolumn{7}{|l|}{ Maio/1992 } \\
\hline & Portaria & 1 & 1 & 0 & 0 & A. darlingi 1 \\
\hline & Cantina & II & 6 & 5 & 0 & A. darlingil \\
\hline & N ível Primário & III & 1 & 1 & 0 & A. darling $\mathrm{i}^{1}$ \\
\hline & Nível Staff/M édio & IV & 0 & 22 & 0 & A. darlingi 1 \\
\hline & Paiol & V & 0 & 0 & 0 & \\
\hline & Planta Sul & VI & 0 & 0 & 0 & \\
\hline & Baixão do Araguary & VII & 4 & - & - & A. darlingi 1 \\
\hline & & & - & 1 & - & A. darlingi 1 \\
\hline & & & - & 1 & - & A. ininii 1 \\
\hline & & & - & - & 2 & A. darlingi 2 \\
\hline & Alojamento Nível Médio & VIII & 0 & 0 & 1 & A. darlingi 3 \\
\hline & Guarita do Lataia & $\mathrm{IX}$ & 0 & 0 & 5 & A. darlingi 3 \\
\hline & Palhoça & $x$ & 0 & 2 & 0 & A. darlingi 3 \\
\hline Total & & & 12 & 32 & 8 & 52 \\
\hline \multicolumn{7}{|c|}{ Agosto/1992 } \\
\hline & Cantina & II & 0 & 1 & 0 & A. darlingi 1 \\
\hline & & & - & 1 & - & A. strodei1 \\
\hline & Nível Primário & III & 0 & 0 & 0 & \\
\hline & Palhoça & $x$ & 0 & 26 & 0 & A. darlingi 1 \\
\hline & & & - & 1 & - & A. $\mathrm{sp} .1$ \\
\hline & Lagoa do Lataia & $X I$ & - & - & 1 & A. darlingi 1 \\
\hline & & & - & - & 6 & A. nuneztovari1 \\
\hline & & & - & - & 5 & A. oswaldoi1 \\
\hline & & & - & - & 1 & A. oswaldoi2 \\
\hline & & & - & - & 1 & A. oswaldoi2 \\
\hline Total & & & 0 & 29 & 14 & 43 \\
\hline \multicolumn{7}{|c|}{ Novembro/1992 } \\
\hline & Cantina & II & 0 & 21 & 0 & A. darlingi 1 \\
\hline & & & 0 & 3 & 0 & A. albitarsis1 \\
\hline & & & 0 & 1 & 0 & A. sp. 1 \\
\hline & Planta Primário & XII & 0 & 2 & 0 & A. darlingi 1 \\
\hline & & & 0 & 2 & 0 & A. albitarsis 1 \\
\hline & & & 0 & 1 & 0 & A. sp.1 \\
\hline & Lagoa do Lataia & $X I$ & 0 & 0 & 67 & A. darlingi 2 \\
\hline & & & 0 & 0 & 4 & A. oswaldoi1 \\
\hline & & & 0 & 0 & 4 & A. sp. 2 \\
\hline & Almoxarifado & XIII & 0 & 0 & 0 & \\
\hline & N ível Staff/M édio & IV & 0 & 0 & 0 & \\
\hline & ETA & XIV & 0 & 2 & 0 & A. darlingi 1 \\
\hline & Palhoça & $x$ & 0 & 19 & 0 & A. darlingi 1 \\
\hline Total & & & 0 & 51 & 75 & 126 \\
\hline Total Geral & & & 12 & 112 & 97 & 221 \\
\hline
\end{tabular}

Fonte: Programa de Saúde da Mineração N ovo Astro S/A.

Ponto = Identificação do local de captura; ETA = Estação de tratamento de água.

1 Captura intra e peridomiciliar com isca humana.

2 Captura extradomiciliar com armadilhas de Shanon e/ou CDC

3 Captura extradomiciliar com isca humana.

O bservador: Téc. R. Lacerda, Fundação Nacional de Saúde, Coordenadoria

Regional do Pará (1992) 
Prevalência de mosquitos infectados capturados na área de abrangência da Mineração Novo Astro $S / A$, no período de maio a novembro de 1992, identificados pelo teste de ELISA, com anticorpos monoclonais* (MAB's) específicos.

\begin{tabular}{|c|c|c|c|c|c|c|}
\hline \multirow[t]{2}{*}{ Período } & \multirow[t]{2}{*}{ Espécie } & \multirow[t]{2}{*}{$\%$} & \multirow[t]{2}{*}{$n$} & \multicolumn{3}{|c|}{ Positividade } \\
\hline & & & & $\mathrm{Pf}$ & PV & Pv-vk247 \\
\hline \multicolumn{7}{|c|}{ Maio/1992 } \\
\hline & A. darlingi & 95,7 & 45 & 0 & 31 & 22 \\
\hline & & & 0 & 22 & - & \\
\hline & A. ininii & 4,3 & 2 & 0 & 0 & 0 \\
\hline Subtotal & & 100,0 & 47 & 0 & $5(10,6 \%)$ & $2(4,3 \%)$ \\
\hline \multicolumn{7}{|c|}{ Agosto/1992 } \\
\hline & A. darlingi & 63,8 & 30 & 0 & 32 & 0 \\
\hline & A. oswaldoi & 14,9 & 7 & 0 & 0 & 0 \\
\hline & A. nuneztovari & 8,6 & 4 & 0 & 0 & 0 \\
\hline & A. strodei & 2,1 & 1 & 0 & 0 & 0 \\
\hline & A. sp. & 10,6 & 5 & 0 & 0 & 0 \\
\hline Subtotal & & 100,0 & 47 & 00 & $3(6,4 \%)$ & 0 \\
\hline \multicolumn{7}{|c|}{ Novembro/1992 } \\
\hline & A. darlingi & 89,5 & 111 & 0 & $5^{1}$ & 0 \\
\hline & A. albitarsis & 4,1 & 5 & 0 & 0 & 0 \\
\hline & A. oswaldoi & 3,2 & 4 & 0 & 0 & 0 \\
\hline & A. sp. & 3,2 & 4 & 0 & 0 & 0 \\
\hline Subtotal & & 100,0 & 124 & 0 & $5(4,0 \%)$ & 0 \\
\hline Total & & & 218 & 0 & 13 & 2 \\
\hline
\end{tabular}

* cedidos por Dr. A. Wirtz (Walter Reed Army Institute of Research, Estados Unidos).

Testes realizados no Laboratório de Entomologia do Programa de Malária, Instituto Evandro Chagas.

$\mathrm{Pf}=$ Plasmo dium falciparum; Pv = Plasmodium vivax.

1 Garimpo Araguary.

2 Lataia, área residencial.

nóstico precoce e tratamento eficaz, associados às medidas seletivas de controle vetorial.

Nos últimos 20 anos, vários autores têm incriminado diferentes espécies de anofelinos como transmissores da malária na região Amazônica brasileira. Além do A. darlingi, que se mantém como transmissor primário, inclusive nas observações do presente trabalho, outras espécies foram consideradas como transmissores secundários ou potencialmente capazes de transmitir a doença. Dixon et al . (1979) em estudos realizados num trecho da rodovia Tranzamazônica, sugerem a possibilidade da participação de outras espécies; Arruda et al. (1986), no Estado do Pará, incrimina o A.oswaldoi, A. trianulatus, A. nuneztovari e A. albitarsis, os três últimos descritos pela primeira vez como transmissores da malária no Brasil; Klein et al. (1991), em Costa Marques, Rondônia, também identificaram o A.al bitarsis como transmissor da malária naquela região; Póvoa (1993), regis- tra a captura de 15 diferentes espécies de mosquitos do gênero Anophel es na região da Serra do Navio, Amapá, além das espécies acima citadas, identifica mais o A. braziliensis e o A. rangeli, este último, positivo somente para $\mathrm{P}$. malariae.

Na medida em que o programa de controle foi sendo implantado, seguindo a seqüência de fases pré-estabelecidas, as correções e/ ou introdução das medidas de control e eram efetivadas como rotina de procedimentos, portanto, ao longo do processo de caracterização do estrato epidemiológico essas medidas já provocavam mudanças no perfil da malária na área da MNA.

Como indicadores para avaliação do impacto epidemiológico causado pelas medidas de controle no âmbito do Programa de Saúde da MNA, podemos ressaltar a evidente redução quantitativa dos casos na população alvo, redução da morbi-mortalidade, evidenciada pela 
diminuição do percentual de casos de malária severa e internações hospitalares; a eficácia dos esquemas terapêuticos aferi da pelo aumento do nível de cura radical com redução drástica dos casos de recrudescência e recaídas; pelos indicadores entomológicos, observando-se a diminuição da densidade anofélica pela quantificação das formas adultas e imaturas nos sítios de vigilância entomológica e, principalmente pela queda do nível de infectividade verificada após a implementação das ações antivetoriais.

Registra-se finalmente, a importância da participação da iniciativa privada no controle da endemia, particularmente aquelas que desenvolvem atividades na região Amazônica brasileira, a exemplo da iniciativa da MNA, no Estado do Amapá aqui reportada, que ao im- plantarem programas específicos de controle da malária, certamente, além de ampliarem o conforto e a produti vidade de seus funcionários e prestadores de serviços, estarão beneficiando, inclusive, a população eventualmente rel acionada às suas atividades, e colaborando para a diminuição da incidência da malária na Amazônia brasileira. Essas ações deverão ser implementadas com orientação e supervisão do próprio MS através de instituições como o IEC, FUNASA, por exemplo, o que garantirá sua exeqüibilidade.

Sugere-se que sejam criados mecanismos oficiais de obrigatoriedade, estimulados por incentivos por parte do governo, às empresas que desenvolvem projetos na região Amazônica brasileira, para implantação de programas específicos de controle da malária.

\section{Agradecimentos}

Os autores agradecem à Dra. Marinete Póvoa, do Instituto Evandro Chagas (IEC) e ao Dr. Inocêncio Gorayebi, do Museu Paraense Emílio Goeldi (MPEG), pela leitura crítica do manuscrito e valiosas sugestões. Agradecem também à Dra. Ana Catarina Xavier Lemos, da Fundação Nacional de Saúde (FUNASA) Amapá, pela provisão de informações e dados estatísticos sobre a malária no Estado e à Dra. Nazaré Segura, do Laboratório de Entomologia - IEC, pela assistência técnica na realização dos testes de ELISA e ao Dr. A. Wirtz, do Walter Reed Army Institute of Research - (Estados Unidos), pelos MAB's cedidos. Finalmente, à Mineração Novo Astro S/A (MNA) pelo suporte logístico e financeiro, bem como aos seus técnicos da área de saúde. 


\section{Referências}

ARRUDA, M.; CARVALHO, M. B.; NUSSENZWEIG, R. S.; M ARACIC, M.; FERREIRA, A. W. \& COCHRANE, A. H., 1986. Potential vectors of malaria and their different susceptibility to Plasmodium falciparum and Plasmodium vivax in northern Brazil identified by immunoassay. American Journal of Tropical Medi cine and Hygiene, 35:873-881.

CALVOSA, V. S.; COUTO, A. A. \& SOUZA, J. M., 1992. Associação modiaquina/tetraciclina no tratamento de malária fal ciparum em pacientes com malária aguda oriund os de áreas com comprovada resistência à cloroquina. In: XXVIII Congresso da Sociedade Brasileira de Medicina Tropical, Resumos, p. 14. Belém: Sociedade Brasileira de Medicina Tropical.

COUTO, A. A.; CALVOSA,V. S.; SANTOS, M. A. \& SOUZA, J. M., 1995. Evolução temporal da resistência in vitro do Plasmodium fal ci parum às drogas antimaláricas em duas áreas da Amazônia brasileira com distintas características sócio-econômicas e geográficas. Revista da Sociedade Brasileira de Medicina Tropical, 28:357-365.

DIXON, K. E.; ROBERTS, D. R. \& LLWELLYN, C. H., 1979. Contribuição ao estudo epidemiológico da malária em trecho da Rodovia Transamazônica, Brasil. Revista do Insti tuto de Medicina Tropical deSão Paulo, 21:287-292.

FUNASA (Fundação Nacional de Saúde), 1996. Dados Epidemiológicos do Estado por Municípios. Macapá: FUNASA.

KLEIN, T. A.; LIMA, J. B. P.;TADA, M. S. \& MILLER, R., 1991. Comparative susceptibility of anopheline mosquitoes in Rondônia, Brazil to infection by Plasmodium vivax. American Journal of Tropical Medicine and Hygiene, 45:463-470.

MARQUES, A. C., 1986. Migration and the dissemination of malaria in Brazil. International Symposium on Malaria. Memórias do Instituto Oswaldo Cruz, 81(Sup. 2):17-30.

MARQUES, A. C., 1987. Human migration and the spread of malaria in Brazil. Parasitology Today, 3:166-170.
MARQUES, A. C.; PINHEIRO, E. A. \& SOUZA, A. G., 1986. Um estudo sobre a dispersão de casos de malária no Brasil. Revista Brasileira de Malariologia eDoenças Tropicais, 38:51-75.

MS (Ministério da Saúde), 1999. Avaliação Epidemiológica da Malária no Brasil. Brasília: Grupo Técnico de Malária, Coordenadoria de Controle de Doenças Transmitidas por Vetores, Departamento de Operações, Fundação Nacional de Saúde, Ministério da Saúde.

OPS (Organizacion Panamericana de la Salud), 1987. Si tuación de la malaria en las Américas, 1996. Boletín Epidemiológico - OPS, 18:1-13.

PINOTTI, M., 1953. Novo método de controle da malária pelo emprego de medicamentos misturados ao sal de cozinha utilizado na alimentação diária. Revista Brasileira deMedicina, 10:241-246.

PÓVOA, M. M., 1993. Studies on Malaria in Serra do Navio Region, Amapa State, Brazil. Ph.D. Thesis, London: Faculty of Medicine, University of London.

TORRESINI, C. A.; GODOY, M. L. S.; COUTO, A. A. \& CALVOSA, V., 1993. Atividades Desenvolvidas pela Mineração Novo Astro S. A., no Estado do Amapá. Macapá: I Encontro de Ciência eTecnologia do Estado do Amapá. (mimeo.)

WIRTZ, R. A.; BURKOT, T. R.; ANDRE, R. G.; ROSENBERG, R.; CILLINS, W. E. \& ROBERTS, D. R., 1985. Identification of Plasmodium vivax sporozoites in mosquitoes using an enzyme-linked immunosorbent assay. American Journal of Tropical Medicine and Hygiene, 34:1048-1054.

WIRTZ, R. A.; ZAVALA, F.; CHAROENVIT, Y.; CAMPBELL, G. H.; BURKOT, T. R.; SCHNEIDER, I.; ESSER, K. M.; BEAUDOIN, R. L. \& ANDRE, R. G., 1987. Comparative testing of monoclonal antibodies against Plasmodi um falci parum sporozoites for ELISA development. Bulletin of theWorld Health Organization, 65:39-45.

WHO (World Health Organization), 1993. Global malaria control. Bulletin of theWorld Health Organization, 7:281-284. 